\title{
PROMs data: can it be used to make decisions for individual patients? A narrative review
}

This article was published in the following Dove Press journal:

Patient Related Outcome Measures

Jonathan Field'

Michelle M Holmes ${ }^{2,3}$

Dave Newell ${ }^{3,4}$

'Back Active, Southsea, Hampshire, UK; ${ }^{2}$ Department of Psychology, University of Southampton, Southampton, Hampshire, UK; ${ }^{3}$ AECC University College,

Bournemouth, Dorset, UK; ${ }^{4}$ Centre for Primary Care and Population Sciences, University of Southampton,

Southampton, Hampshire, UK
Correspondence: Jonathan Field Back Active, Southsea, Hampshire PO4 ODN, UK

Tel +44I730776763

Email jonathan@b2haqp.co.uk
Abstract: Patient-reported outcome measures (PROMs) are increasingly used in clinical practice providing health care professionals with patients' perceptions and views of their health. They have traditionally been utilized in health research and health service evaluation and are now starting to be used in routine clinical practice with individual patients. The repeated administration of PROMs over the course of care with individual patients has a role in patient assessment, assisting clinical decision-making, and tracking patient progress. This approach can influence the patient-clinician encounter impacting the therapeutic alliance and increasing patient engagement with care. It is also theorized to improve patient outcomes and satisfaction with care. Advances in technologies and innovations in methodology have led to the use of electronic systems to simplify the collection and reporting of PROMs. Challenges of using PROMs with individual patients include clinician knowledge and skills, and access to appropriate technology. This paper reviews the use of PROMs with individual patients, illustrating how they may affect the patient-clinician encounter impact satisfaction and health outcomes. The routine use of PROMs during a course of care rather than just at the start and end provides additional opportunity to inform clinician and patient with benefits to both. The adoption of PROMs in clinical practice can help health care professionals to make decisions for individual patients. Further work is needed to examine the implementation of PROMs and benefits of PROMs in different clinical contexts.

Keywords: patient-reported outcome measures, PROMs, patient outcome assessment, clinical decision-making

\section{Introduction}

Patient-reported outcome measures (PROMs) are a form of validated self-report instruments which use patients' views to assess their health status and well-being. ${ }^{1}$ These standardized tools capture patients' views, feelings and experiences and can measure changes in health status and quality of life. ${ }^{1,2}$

The use of outcome measures has been incorporated into clinical practice with patients' subjective views deemed as valuable information to evaluate health care as well as assessing the efficacy of conventional medical treatment. ${ }^{3}$ In the early 1990s, PROMs had three main uses within clinical practice: increase knowledge of disease trajectories, evaluate the effectiveness of treatment, and assess the quality of the care provided. ${ }^{3}$ These outcomes were suggested to be intrinsically linked to processes of providing quality health care. ${ }^{4-6}$

PROMs are increasingly being routinely used across a range of health services to improve care. By measuring aspects of health that are important to patients. They aid the provision of patient-centered health care, and their collection helps demonstrate to external observers that patient experiences are considered significant. ${ }^{7}$ 
PROMs are well-established in their use in research to evaluate the impact of treatment. ${ }^{8}$ Increasingly, the data from PROMs are used to provide an insight into the effectiveness, appropriateness and acceptability of medical, surgical and therapeutic technologies, as well as the impact of these interventions on patients' physical or psychological symptoms, disability, functioning and overall quality of life. ${ }^{6}$ Additionally, PROMs have a role in health care evaluation where they are increasingly utilized in routine clinical practice as an effective way to evaluate the quality of a provided service. Aggregated PROM data from individual patients can be used for audit, with the data utilized to examine the impact, appropriateness, quality and performance of health care services. ${ }^{6,9,10}$ This can be collected internally within institutions or externally as part of mandated data reporting. The collection of outcome data is seen to place positive pressure on health care providers to respond and improve patient care. ${ }^{8}$ Within the UK National Health Service (NHS), routine measurement has been called for to benchmark performance of health care services ${ }^{9}$; with certain areas of the NHS requiring health care providers to invite patients to complete pre-operative and post-operative PROMs questionnaires.

Repeated administration of PROMs may be used in routine clinical practice at the individual patient level during a course of care. This approach to using PROMs can aid clinicians' provision of care for patients, by presenting patients' views of their health which can complement the traditional method of medical history acquisition and physical examination. This provision of information can facilitate patient-centered care and assist in shared decision-making. However, PROMs are infrequently collected in routine clinical practice in a standardized manner for use at individual patient level. ${ }^{8}$

In this article, we will discuss the use of PROMs with individual patients and their utilization throughout a patients' course of care. This review outlines the potential benefits of using PROMs in the care of individual patients, innovative methods in the provision of PROMs, and the current challenges to implementation.

\section{The use of PROMs for individual patients}

\section{Assessment}

Patients may provide "baseline" data by completing PROM questionnaires either before their appointment or within their first-visit alongside their clinician. This information can contribute to the initial assessment, alongside the case histories and patient examination. ${ }^{11}$ Clinicians may use PROMs related to a patient's specific condition, or more general ones ascertain a baseline of the patient's health-related quality-oflife, functioning, or well-being. ${ }^{12}$ They may help clinicians detect underlying issues. For example, patients may present with a range of multi-faceted issues, but only discuss one with their clinician. PROMs responses potentially allowing clinicians to identify issues that may have previously been overlooked, ${ }^{13,14}$ or from another domain, such as their physical, emotional, or social functioning. ${ }^{15}$

Improvements in health and changes in lifestyles are impacting the longevity of many populations. However, success in mortality reduction is not matched by improvements in the level of morbidity with people aging while suffering from multiple and more complex conditions. In relation to this, PROMs can enable a patient to report as to which aspect of their care is of main concern to them. This allows clinicians and patients to identify issues and prioritize any concerns appropriately within the treatment. ${ }^{12,13,16,17}$

Traditional models for health care delivery have rewarded providers based upon activity; as the demand and cost of health care rise, it is argued that it is value, rather than the amount of care, which is considered most important. ${ }^{18}$ Value-based health care is a mechanism to help focus resources where they may have the largest impact, and the use of PROMs to help explicitly prioritize health outcomes that matter to patients relative to the cost of achieving those outcomes is considered fundamental in this.

Patient-reported questionnaires may also be used for screening. ${ }^{19}$ Including checking for a particular condition, ${ }^{16,20}$ for example, depression or anxiety, or alerting clinicians to a problem or issue not previously mentioned by the patient. ${ }^{15,21}$ In a systematic review, exploring the effectiveness of providing feedback on PROMs to clinicians, Espallargues et al found that PROMs influenced the assessment of a patient through its screening action and this had a positive effect on diagnosis. ${ }^{22}$ Clinicians may also use PROMs to monitor disease progression, ${ }^{17}$ whether by assessing the severity of a symptom or the change in functioning, augmenting a clinicians awareness of changes or progression. ${ }^{23-25}$

\section{Clinical decision-making}

Using PROMs helps to manage patient perspectives, by facilitating communication between patients and clinicians. ${ }^{19,26}$ The information provided by PROMs can act as a forum for discussion between caregiver and patients on issues of concern to the patient. Through exploring 
PROM responses of clinicians may gain insight from the patient perspective. ${ }^{27}$ This increased awareness of factors considered significant to patients is thought to be useful in guiding treatment planning in clinical settings. ${ }^{3,13,28}$ It is suggested the extra-information revealed in PROMs may encourage clinicians to have a more holistic view of the patient, focusing on patients' views in addition to the results of biophysical measures and clinical tests. ${ }^{12,29}$

Having access to the patient perspective as revealed in PROMs assists clinicians in providing patient-centered care. $^{29,30}$ Used within the clinical encounter, PROMs provide an opportunity for clinicians to discuss patient expectations of treatment, and potentially identify unrealistic expectations. $^{31}$ This allows patients and clinicians to establish shared and realistic treatment goals and taking into account patient preferences. ${ }^{32-34}$

In providing information while completing PROMs patients can be motivated to start up a dialogue about their care and treatment options thus feeling empowered to be in the decision-making process. ${ }^{27}$ By bringing awareness of patient's desired outcomes and treatment goal to the clinician they can provide insight into patients perception of their health. ${ }^{35}$

In terms of improvement, it can be difficult for patients to remember initial symptomatology severity or be aware of change over time. In this regard. It is reported that by completing PROMs at various time points during their treatment and having access to the results helps individuals in identifying improvements in their health. ${ }^{31}$ This awareness assists patients with decisions to continue with a particular care or seek change can motivate them towards compliance with health advice and has an effect on their satisfaction with treatment.

Several PROMs have been produced specifically to assist with clinical decision-making. For example, the Orebro Musculoskeletal Pain Screening Tool has established thresholds suggestive for providing patients with cognitive behavioral approaches alongside manual therapy $^{36}$ while the more recent STarT Back tool has robust evidence for providing improved patients outcomes and cost savings through its ability to assist clinicians to triage patients to one of the three treatment pathways. ${ }^{37}$

\section{Tracking progress and evaluating treatment}

PROMs can be used to monitor patients' conditions during a course of care, for example, severity or frequency of symptoms, or functioning. ${ }^{22,25,38}$ Information from this monitoring can aid clinicians' appreciation of how a patient is doing with a treatment and assist regarding decisions on modifying or changing components of care. ${ }^{39,40}$ Furthermore, this may aid decisions around ordering further tests and onward referral of patients. ${ }^{26,27,33}$

For those presenting with complex conditions such as mental health issues or pain conditions, it is not unusual in clinical settings for a range of therapeutic approaches to be incorporated into each treatment session, with clinicians tailoring the treatment to meet individual patient's needs. ${ }^{41}$ Health care professionals select the components of each clinical encounter based upon their understanding of efficacy demonstrated through trials, personal clinical experience, and patient preference. Their choice to continue or change elements of care will also be informed by changes in health status and other factors including the therapeutic relationship between clinician and the person receiving care. For example, in populations attending for chiropractic care, lack of change in presenting symptoms soon after starting care is linked to a poor eventual prognosis. Being able to identify lack of progress is important and PROMs administered during a course of care may support this. ${ }^{42-44}$ Similarly, this has been demonstrated in psychological services, where outcomes have been shown to improve if practitioners are given real-time outcome data to identify failing patients enable them to amend the care provided. ${ }^{45}$ PROM reports during care are felt helpful in assisting practitioners by providing the information needed to confidently continue or alter the care they are providing. ${ }^{46}$

\section{Potential benefits of using PROMs for individual patients Improving the patient-clinician encounter} Theoretically, using PROMs with individual patients may improve health care delivery by impacting on patientclinician interactions. PROMs are proposed to have a cascading effect, with their information raising awareness of symptoms to both patients and clinicians, facilitating a discussion during clinical encounters. ${ }^{27}$

Clinicians view the use of PROMs with individual patients as potentially impacting on the processes of care, such as influencing communication, shared decision-making and planning care. ${ }^{21}$ In a systematic review, Boyce et al found that PROMs can provide a prompt for discussion, facilitating communication between the patient and clinician. $^{19,23}$ Patients completing PROM assessments have described how discussions they help open focus on 
issues considered important. ${ }^{47}$ This focusing of the discussion through the identification of problems may reduce the number of questions to be asked by the clinician, shortening the patient history examination and leaving more time for treatment or discussion of treatment options.

Awareness of patients' needs can be raised by use of PROM both at initial assessment and ongoing monitoring throughout a course of care. ${ }^{17}$ The information available from PROMs enable both the clinician and patient to identify and prioritize key patient issues, with PROMs providing information on what is the most troublesome or the biggest priority for treatment. Increased communication on patients' issues or concerns allows clinicians to identify any patient education need and prescribe specific support and tailored education or counseling. ${ }^{33}$ This improved patient-centered communication may contribute to greater patient satisfaction with care. ${ }^{13}$

The use of PROMs is also thought to influence the therapeutic relationship between patients and clinicians. By providing information, and encouraging discussion, patients perceive clinicians have a deeper understanding of their condition and their experiences. ${ }^{21}$ In an age of increasing reliance on technology in patient assessment and monitoring, asking patients' views and listening to their perceptions of their health status and progress has been described as 'humanizing care. ${ }^{48}$

\section{Improvements in patients' self- management of their condition}

PROMs may improve an individuals' understanding of their condition. Through this increased knowledge and in conjunction with ongoing feedback through monitoring, perceived control of health may be enhanced. In older patients, this feeling of control is seen to feed through to further feelings of empowerment in the clinical encounter. ${ }^{49}$

Largely through the use of electronically delivered PROM (ePROM), patients may record and track changes in their health status at home. Doing this, outside clinical encounters may improve patients' engagement and selfmanagement of their condition between appointments. ${ }^{27}$ This may include decisions on treatment choices such as timing of visits to health care professionals. Ongoing monitoring of conditions increases patient compliance to treatment plans and self-management advice from clinicians. In a randomized control study of people undergoing chemotherapy it was found that those able to report symptoms to the hospital remotely and receiving back self-care information based on these, had improved management of symptoms and felt reassured by the level of support ${ }^{50}$ Early identification of deterioration of symptoms in patients undergoing radiotherapy for lung cancer through self-reported home monitoring produced improvements in anxiety, drowsiness and self-care self-efficacy. ${ }^{51}$

\section{Innovations in use of PROMs for individualized patients \\ The use of individualized PROMs}

It may be argued that using preset questionnaires when assessing patients' health status introduces limitations if one of the goals of PROMs is to capture the elements of care that are important to patients. Several measures have been developed which encourage patients to indicate the specific issues they want to address, and these are used as items within the measurement tool. These individualized PROMs (I-PROMs) have been found to be useful in improving communication during consultation with general medical practitioners, psychologists as well as complementary therapists in particular increasing attention to patients' preferences. ${ }^{52,53}$ Ashworth et al. ${ }^{54}$ reported that over half of patients provided novel and relevant clinical information in their free-text responses when completing an I-PROM that would otherwise not have been considered in their outcome assessment.

A disadvantage of I-PROMs is that as each patient is effectively writing their own questionnaire there is some doubt as to the validity of using aggregated results from groups of patients for clinical audit or service evaluation.

\section{Electronic measurement systems to support PROMs for individual patient care}

Electronic measurement systems are an innovative method to implement PROMs throughout a patient's course of care. These systems may be delivered via a variety of methods, including: web-based systems, email reminders, and mobile applications. ${ }^{55,56}$ The use of mobile applications to collect electronic PROMs (ePROM) in a clinical setting is still in infancy. Early concerns in using mobile devices to collect patient-reported data centered on potential lack of access to mobile devices, particularly in older age groups. A recent report estimated that in 2012, around $52 \%$ of the survey group owned such a device, while in $2017,85 \%$ of respondents said that they own such a device. ${ }^{57}$ In 2017 , the proportion of those owning a smart phone between the 
ages of 16 and 24 was $96 \%$. However, while over $80 \%$ of those between 35 and 54 years of age owned a smart phone, this proportion was only around $50 \%$ in the 55-64-year-old bracket. ${ }^{58}$ While, caution must be exercised when assuming that older age groups have equal access to such devices, particularly in the light that such a demographic has a higher incidence of chronic conditions that may require monitoring by PROMs, the extensive penetration of mobile device use within the adult population at least up to age of 55 suggests that smart phone ownership is unlikely to be a barrier to the use of apps for PROM collection. ${ }^{59}$

The literature around the use of mobile apps to record PROMs is limited and heterogeneous with the focus of work centering on the equivalence of ePROMs to paper versions. A recent trial exploring the willingness of patients in an equivalence clinical trial to download an app to record patient-reported outcomes found high associations between scales presented using a paper format, a provided device and the patient's own device. Of the 155 subjects involved, 95\% stated they would be willing to download an app on their own devices to report PROMs in a trial setting. ${ }^{60}$ An earlier Cochrane review also found extensive equivalence between surveys collected using apps and mobile devices and paper versions. ${ }^{61}$ The results suggest that equivalence was not compromised with the use of apps and that the use of apps may shorten completion times in comparison to paper and SMS. The authors conclude that apps might not affect data equivalence given the use of the questionnaire, frequency and intended setting were matched to the questionnaires' original intentions. ${ }^{61}$ Although many studies included in the review explore aspects of acceptability to patients the results were inconclusive overall.

The collection of PROMs during treatment and management of cancer has also been explored using mobile apps. ${ }^{62}$ One clear advantage in such pathological conditions is the potential to uncover changes in symptomatology early on with the requisite opportunity to intervene or modify treatment approaches. One such study looked at patient reporting of side effects and symptomatology during radiation treatment for head and neck cancers and reported that the median compliance among 55 patients was $71 \%$, concluding that such methods of reporting were potentially effective ways to avoid escalation of symptoms and avoid hospital admissions. ${ }^{63}$

Finally, the potential of "bring your own device" approaches of collecting ePROMs during clinical trials in the patients' own environment has been viewed as potentially revolutionizing the monitoring of patients on a periodic or ongoing basis. Such an approach is thought to address the practical and cost concerns of providing patients with standalone devices which can be easily duplicated in app form downloaded to the patient's smart device. However, the potentially bright future of using such approaches within clinical trials and beyond in routine clinical practice also come with limitations and considerations. Health care providers must decide between the use of web-based applications (ie, web pages accessed through a browser on the phone) as opposed to native applications (apps); stand-alone software on the smart device itself can bring both advantages and disadvantages. For example, native applications are normally built as stand-alone apps and can incur greater time and costs compared to a web-based system. ${ }^{64}$ However, this approach in trials shows compliance using ePROM collection methods on smart devices can be very high. ${ }^{65}$

With the advent of the "quantified self" and the spread of ever more tracking and monitoring devices capable of measuring health parameters on a day to day basis, there is increased focus on the use of such devices and software applications for clinical practice. ${ }^{41}$ The future of mobile applications as a method for using PROMs with individual patients appears the next logical step with increasing expectation and acceptance on the part of clinicians and patients as the use of smart devices becomes ever more pervasive in modern life.

\section{Implementation of PROMs for individual patients Selecting PROMs}

The implementation of routine data collection for individual patients must consider the appropriate PROM instrument. In implementing PROMs, it is important that both patients and clinicians are involved in the selection of important outcomes to measure. ${ }^{66}$ The PROMs must be clinically relevant and applicable to routine clinical practice for clinicians engagement ${ }^{67}$ The concepts measured must be important and meaningful to patients, and may include intensity of symptoms, quality of life, or activity limitations. ${ }^{29}$ Additionally, PROMs must be validated demonstrating their suitability for routine clinical practice, including reliability, validity, responsiveness, and acceptability to patients. ${ }^{52}$

Using PROMs with individual patients, additional assessments rather than pre- and post-treatment may be necessary, so clinicians must consider the timing of measurements and the frequency of asking patients to complete PROMs. ${ }^{68}$ An 
additional challenge is considering the intensity of administration and the complexity of questionnaires, as these impact on the potential burden for patients which should be balanced against the usefulness for clinical practice. ${ }^{69,70}$

\section{Clinician knowledge and engagement}

The use of PROMs with individual patients is only possible with active clinician engagement and willingness to integrate their use into routine clinical practice. Clinicians' lack of knowledge and education surrounding PROMs is a significant barrier to PROM use. ${ }^{67,71}$ If electronic systems are used, clinicians must feel confident with using the software, as unfamiliarity with electronic PROM software is a barrier to successful implementation. ${ }^{28}$ Training education and support for clinicians should include the administration of electronic systems, the purposes of PROMs, and how to interpret data, and their potential benefits. ${ }^{66,71,72}$ In qualitative interviews with chiropractors, clinicians often had a lack of knowledge and engagement with PROMs, but were positive about training which could improve their knowledge and engagement through PROMs with individual patients. ${ }^{46}$

\section{Technology}

The practicalities of collecting PROMs to be fed back to clinicians within the consultation can be challenging with administrative time affecting workflow ${ }^{8}$ Electronic PROMs are recommended for making their data more accessible within clinics and reducing the time-burden. Electronic systems for PROMs are acceptable to patients. ${ }^{70}$ Patients' perceive web-based PROMs as easy, and are willing to complete PROMs at home ${ }^{73-75}$ Despite concerns that older patients will have difficulties in using technology, studies report they are able to use systems with little difficulty. ${ }^{74,75}$

Clinicians require a certain level of technology to implement PROMs that be used routinely with individual patients, including the appropriate hardware, software, and internet access. $^{76}$ Any electronic system must be conducive to use within clinical settings, with PROMs presented in a format that is easy for the patient to navigate and complete, and provide data in a format that is easily accessible for the clinician. $^{70,77}$

\section{Conclusion}

PROMs are increasingly used in clinical practice, capturing patients' views on their health status and well-being. PROMs are used to evaluate health service provision, assess the impact or efficacy of treatment, and the quality of care for individual patients. PROMs collected in routine clinical practice in a standardized manner at individual patient level can aid clinicians' provision of care for patients. The information generated by PROMs can be used in the initial assessment to help incorporate patients' perceptions and views about their health into clinicians understanding of the patients' needs. They also can facilitate patient-centered care through clinical decisionmaking and have a role in promoting shared decision-making. Through their ability to monitor health status over time, PROMs can be used in the tracking of patient progress. Increasing awareness of changes in patients' health status allows clinicians to evaluate treatment, with the data provided helping clinicians in decisions regarding changing or continuing elements of a patients care. This paper has briefly reviewed the potential benefits of this approach to using PROMs, illustrating how PROMs may impact the patient-clinician encounter-improving satisfaction and impacting health outcomes. The routine use of PROMs during a course of care rather than just at the start and end provides additional opportunity to inform clinician and patient with benefits to both.

In real-world settings, the development and adoption of technology should be encouraged by health care professionals as an aid to successful implementation of PROM collection and the presentation of their results.

A focus on implementation research is needed to overcome some of the challenges of using PROMs. Further research is also required to understand implementation strategies in different clinical contexts.

In conclusion, adoption of PROMs in clinical practice can help health care professionals to make decisions for individual patients. By measuring aspects of health that are important to patients, PROMs can inform clinicians about health management and treatment plans, aiding the provision of patientcentered health care.

\section{Disclosure}

Dr Jonathan Field has an interest in the Care Response ePROM system. This is a free to use product which does not have any financial benefit. The authors report no other conflicts of interest in this work.

\section{References}

1. Dawson J, Doll H, Fitzpatrick R, Jenkinson C, Carr AJ. The routine use of patient reported outcome measures in healthcare settings. $B M J$. 2010;340:c186. doi:10.1136/bmj.c293

2. Bausewein C, Daveson B, Benalia H, Simon ST, Higginson IJ. Outcome Measurement in Palliative Care the Essentials. UK: PRISMA; 2011.

3. Greenfield S, Nelson EC. Recent developments and future issues in the use of health status assessment measures in clinical settings. Med Care. 1992;30(5 Suppl):Ms23-Ms41. 
4. Nelson EC, Berwick DM. The measurement of health status in clinical practice. Med Care. 1989;27(3 Suppl.):S77-S90.

5. Wilson IB, Cleary PD. Linking clinical variables with health-related quality of life: a conceptual model of patient outcomes. JAMA. 1995;273 (1):59-65.

6. National Institute for Clinical Excellence. Guide to the Methods of Technology Appraisal. London: NICE; 2004.

7. Calvert M, Kyte D, Price G, Valderas JM, Hjollund NH. Maximising the impact of patient reported outcome assessment for patients and society. BMJ. 2019;364:k5267. doi:10.1136/bmj.142

8. Greenhalgh J, Dalkin S, Gooding K, et al. Functionality and feedback: a realist synthesis of the collation, interpretation and utilisation of patient-reported outcome measures data to improve patient care. Health Serv Deliv Res. 2017;5(2).

9. Department of Health. Guidance on the Routine Collection of Patient Reported Outcome Measures (PROMs). UK; 2008. Available from: http://www.healthcare-today.co.uk/doclibrary/docu ments/pdf/152_guidance_on_routine_collection.pdf. Accessed July 22, 2019 .

10. Appleby J, Devlin NJ. Measuring NHS Success: Can Patients'Views on Health Outcomes Help to Manage Performance? London: King's Fund; 2005.

11. Osoba D, Translating the science of patient-reported outcomes assessment into clinical practice. J National Cancer Inst Monogr. 2007;37:5-11. doi:10.1093/jncimonographs/lgm002

12. Fung $\mathrm{CH}$, Hays $\mathrm{RD}$. Prospects and challenges in using patientreported outcomes in clinical practice. Qual Life Res. 2008;17 (10):1297-1302. doi:10.1007/s11136-008-9379-5

13. Higginson IJ, Carr AJ. Measuring quality of life: using quality of life measures in the clinical setting. BMJ (Clin Res Ed). 2001;322 (7297):1297-1300. doi:10.1136/bmj.322.7297.1297

14. Valderas JM, Kotzeva A, Espallargues M, et al. The impact of measuring patient-reported outcomes in clinical practice: a systematic review of the literature. Qual Life Res. 2008;17(2):179-193. doi:10.1007/s11136-007-9295-0

15. Greenhalgh J. The applications of PROs in clinical practice: what are they, do they work, and why? Qual Life Res. 2009;18(1):115-123. doi:10.1007/s11136-008-9430-6

16. Snyder CF, Aaronson NK. Use of patient-reported outcomes in clinical practice. Lancet. 2009;374(9687):369-370. doi:10.1016/S01406736(09)61400-8

17. Lohr KN, Zebrack BJ. Using patient-reported outcomes in clinical practice: challenges and opportunities. Qual Life Res. 2009;18(1):99107. doi:10.1007/s11136-008-9413-7

18. Economist Intelligence Unit. Value-based healthcare: a global assessment. 2016; Available from: http://vbhcglobalassessment.eiu.com/ wp-content/uploads/sites/27/2016/09/EIU_Medtronic_Findings-andMethodology.pdf. Accessed July 7, 2019

19. Boyce MB, Browne JP. Does providing feedback on patient-reported outcomes to healthcare professionals result in better outcomes for patients? A systematic review. Qual Life Res. 2013;22(9):2265-2278. doi:10.1007/s11136-013-0390-0

20. Snyder CF, Jensen RE, Segal JB, Wu AW. Patient-reported outcomes (PROs): putting the patient perspective in patient-centered outcomes research. Med Care. 2013;51(8 Suppl 3):S73-S79. doi:10.1097/ MLR.0b013e31829b1d84

21. Boyce MB, Browne JP, Greenhalgh J. The experiences of professionals with using information from patient-reported outcome measures to improve the quality of healthcare: a systematic review of qualitative research. BMJ Qual Saf. 2014;23(6):508-518. doi:10.1136/bmjqs-2013-002524

22. Espallargues M, Valderas JM, Alonso J. Provision of feedback on perceived health status to health care professionals: a systematic review of its impact. Med Care. 2000;38(2):175-186.
23. Greenhalgh J, Meadows K. The effectiveness of the use of patientbased measures of health in routine practice in improving the process and outcomes of patient care: a literature review. J Eval Clin Pract. 1999;5(4):401-416.

24. Fitzpatrick RM, Fletcher A, Gore S, Jones D, Spiegelhalter D, Cox D. Quality of life measures in health care. I: Applications and issues in assessment. BMJ (Clini Res Ed). 1992;305(6861):1074-1077. doi:10.1136/bmj.305.6861.1074

25. Coon CD, McLeod LD. Patient-reported outcomes: current perspectives and future directions. Clin Ther. 2013;35(4):399-401. doi:10.1016/j.clinthera.2013.01.012

26. Detmar SB. Use of HRQOL questionnaires to facilitate patient-physician communication. Expert Rev Pharmacoecon Outcomes Res. 2003;3(3):215-217. doi:10.1586/14737167.3.3.215

27. Santana M-J, Feeny D. Framework to assess the effects of using patientreported outcome measures in chronic care management. Qual Life Res. 2014;23(5):1505-1513. doi:10.1007/s11136-013-0596-1

28. Chang $\mathrm{CH}$. Patient-reported outcomes measurement and management with innovative methodologies and technologies. Qual Life Res. 2007;16(Suppl. 1):157-166. doi:10.1007/s11136-007-9196-2

29. Snyder CF, Aaronson NK, Choucair AK, et al. Implementing patientreported outcomes assessment in clinical practice: a review of the options and considerations. Qual Life Res. 2012;21(8):1305-1314. doi:10.1007/s11136-011-0054-x

30. Black N. Patient reported outcome measures could help transform healthcare. BMJ (Online). 2013;346:7896.

31. Wright JG. Evaluating the outcome of treatment. Shouldn't We be asking patients if they are better? J Clin Epidemiol. 2000;53(6):549-553.

32. Bitton A, Onega T, Tosteson ANA, Haas JS. Toward a better understanding of patient-reported outcomes in clinical practice. $A m J$ Manag Care. 2014;20(4):281-283.

33. Greenhalgh J, Long AF, Flynn R. The use of patient reported outcome measures in routine clinical practice: lack of impact or lack of theory? Soc Sci Med. 2005;60(4):833-843. doi:10.1016/j.socscimed.2004.06.022

34. Marshall S, Haywood K, Fitzpatrick R. Impact of patient-reported outcome measures on routine practice: a structured review. $J$ Eval Clin Pract. 2006;12(5):559-568. doi:10.1111/j.13652753.2006.00650.x

35. Carver CS, Scheier MF. Control theory: A useful conceptual framework for personality-social, clinical, and health psychology. Psychol Bull. 1982;92(1):111. doi:10.1037/0033-2909.92.1.111

36. Linton SJ, Halldén K. Can we screen for problematic back pain? A screening questionnaire for predicting outcome in acute and subacute back pain. Clin J Pain. 1998;14(3):209-215.

37. Hill JC, Whitehurst DG, Lewis M, et al. Comparison of stratified primary care management for low back pain with current best practice (STarT Back): a randomised controlled trial. Lancet. 2011;378 (9802):1560-1571. doi:10.1016/S0140-6736(11)60937-9

38. Valderas JM, Alonso J, Guyatt GH. Measuring patient-reported outcomes: moving from clinical trials into clinical practice. Med $J$ Aust. 2008;189(2):93-94.

39. Lewis S. Realizing the PROMise of PROMs. Healthcarepapers. 2011;11(4):20-23.

40. Palfreyman S. Patient-reported outcome measures and how they are used. Nurs Older People. 2011;23(1):31-36. doi:10.7748/ nop2011.02.23.1.31.c8295

41. Swan MJJopm. Health 2050: the realization of personalized medicine through crowdsourcing, the quantified self, and the participatory biocitizen. J Pers Med. 2012;2(3):93-118.

42. Axén I, Jones JJ, Rosenbaum A, et al. The nordic back pain subpopulation program: validation and improvement of a predictive model for treatment outcome in patients with low back pain receiving chiropractic treatment. J Manipulative Physiol Ther. 2005;28(6):381385. doi:10.1016/j.jmpt.2005.06.008 
43. Malmqvist S, Leboeuf-Yde C, Ahola T, et al. The nordic back pain subpopulation program: predicting outcome among chiropractic patients in Finland. Chiropr Osteopat. 2008;16(1):13.

44. Newell D, Field J. Who will get better? Predicting clinical outcomes in a chiropractic practice. Clinical Chiropractic. 2007;10(4):179-186.

45. Newnham EA, Page A. Bridging the gap between best evidence and best practice in mental health. Clin Psychol Rev. 2010;30(1):127-142.

46. Holmes MM, Bishop FL, Newell D, Field J, Lewith G. Chiropractors' views on the use of patient-reported outcome measures in clinical practice: a qualitative study. Chiropr Man Therap. 2018;26(1):50. doi:10.1186/s12998-018-0219-6

47. Hvitfeldt H, Carli C, Nelson EC, Mortenson DM, Ruppert BA, Lindblad S. Feed forward systems for patient participation and provider support: adoption results from the original US context to Sweden and beyond. Qual Manage Healthcare. 2009;18(4):247256. doi:10.1097/QMH.0b013e3181bee32e

48. Bottega FH, Fontana RT. Pain as the fifth vital sign: use of the assessment scale by nurses in general hospital. Text Context Enfermagem. 2010;19(2):283-290. doi:10.1590/S0104-07072010000200009

49. Feldman-Stewart D, Brundage MD. A conceptual framework for patient-provider communication: a tool in the PRO research tool box. Qual Life Res. 2009;18(1):109-114. doi:10.1007/s11136-008-9417-3

50. Kearney N, McCann L, Norrie J, et al. Evaluation of a mobile phonebased, advanced symptom management system (ASyMS(C) in the management of chemotherapy-related toxicity. Supportive Care Cancer. 2009;17(4):437-444. doi:10.1007/s00520-008-0515-0

51. Maguire R, Ream E, Richardson A, et al. Development of a novel remote patient monitoring system: the advanced symptom management system for radiotherapy to improve the symptom experience of patients with lung cancer receiving radiotherapy. Cancer Nurs. 2015;38(2):E37-E47. doi:10.1097/NCC.0000000000000150

52. Fitzpatrick RM, Davey C, Buxton MJ, Jones DR. Evaluating patientbased outcome measures for use in clinical trials. Health Technol Assess (Rockv). 1998;2(14). doi:10.3310/hta2140

53. Paterson C. Measuring outcomes in primary care: a patient generated measure, MYMOP, compared with the SF-36 health survey. BMJ. 1996;312(7037):1016-1020. doi:10.1136/bmj.312.7037.1016

54. Ashworth M, Robinson S, Evans C, Shepherd M, Conolly A, Rowlands G. What does an idiographic measure (PSYCHLOPS) tell us about the spectrum of psychological issues and scores on a nomothetic measure (CORE-OM)?. Prim Care Community Psychiatr. 2007.12(1):7-11

55. Fortner B. The challenge of electronically captured patient-reported outcomes. J Oncol Pract. 2007;3(3):120. doi:10.1200/JOP.0731503

56. Jensen RE, Snyder CF, Abernethy AP, et al. Review of electronic patient-reported outcomes systems used in cancer clinical care. $J$ Oncol Pract. 2013;10(4):e215-e222. doi:10.1200/JOP.2013.001067

57. Consultancy United Kingdom. UK smartphone penetration continues to rise to $85 \%$ of adult population. 2017; Available from: https:// www.consultancy.uk/news/14113/uk-smartphone-penetration-con tinues-to-rise-to-85-of-adult-population. Accessed May 172018.

58. Statistica. UK: smartphone ownership by age from 2012-2017. 2017. Accessed May 17, 2018.

59. Kim BY, JJJm L. Smart devices for older adults managing chronic disease: a scoping review. JMIR Mhealth Uhealth. 2017;5(5):82-85.

60. Byrom B, Doll H, Muehlhausen W, et al. Measurement equivalence of patient-reported outcome measure response scale types collected using bring your own device compared to paper and a provisioned device: results of a randomized equivalence trial. Value Health. 2018;21(5):581-589. doi:10.1016/j.jval.2017.10.008
61. Belisario JSM, Jamsek J, Huckvale K, O’Donoghue J, Morrison CP, Car J. Comparison of Self-administered Survey Questionnaire Responses Collected Using Mobile Apps versus Other Methods. Cochrane Database of Systematic Reviews. 2015(7). doi:10.1002/14651858. MR000042.pub2

62. Jensen RE, Snyder CF, Abernethy AP, et al. Review of electronic patient-reported outcomes systems used in cancer clinical care. $J$ Oncol Pract. 2014;10(4):e215-e222. doi:10.1200/JOP.2013.001067

63. Falchook AD, Tracton G, Stravers L, et al. Use of mobile device technology to continuously collect patient-reported symptoms during radiation therapy for head and neck cancer: a prospective feasibility study. Adv Radiat Oncol. 2016;1(2):115-121.

64. Gwaltney C, Coons SJ, O’Donohoe P, et al. "Bring Your Own Device" (BYOD): the future of field-based patient-reported outcome data collection in clinical trials? Ther Innov Regul Sci. 2015;49(6):783-791.

65. Hufford, Michael \& Shields, Alan. (2002). Electronic diaries: Applications and what works in the field. Applied Clinical Trials. 11. 46-56.

66. Callaly T, Hallebone EL. Introducing the routine use of outcomes measurement to mental health services. Aust Health Rev. 2001;24(1):43-50.

67. Duncan EA, Murray J. The barriers and facilitators to routine outcome measurement by allied health professionals in practice: a systematic review. BMC Health Serv Res. 2012;12(1):96. doi:10.1186/ 1472-6963-12-96

68. Jongen PJ, Sanders E, Zwanikken C, et al. Adherence to monthly online self-assessments for short-term monitoring: a 1-year study in relapsingremitting multiple sclerosis patients after start of disease modifying treatment. Patient Prefer Adherence. 2013;7:293. doi:10.2147/PPA.S40173

69. Philpot LM, Barnes SA, Brown RM, et al. Barriers and benefits to the use of patient-reported outcome measures in routine clinical care: a qualitative study. Am J Med Qual. 2018;33(4):359-364. doi:10.1177/ 1062860617745986

70. Gilbert A, Sebag-Montefiore D, Davidson S, Velikova G. Use of patient-reported outcomes to measure symptoms and health related quality of life in the clinic. Gynecol Oncol. 2015;136(3):429-439. doi:10.1016/j.ygyno.2014.11.071

71. Antunes B, Harding R, Higginson IJ. Implementing patient-reported outcome measures in palliative care clinical practice: a systematic review of facilitators and barriers. Palliat Med. 2014;28(2):158-175. doi:10.1177/0269216313491619

72. Santana MJ, Haverman L, Absolom K, et al. Training clinicians in how to use patient-reported outcome measures in routine clinical practice. Qual Life Res. 2015;24(7):1707-1718. doi:10.1007/s11136-014-0903-5

73. Koevoets R, de Glas NA, Le Bourlout C, et al. Autonomous online health assessment questionnaire registry in daily clinical practice. Rheumatology. 2013;52(5):883-887. doi:10.1093/rheumatology/kes389

74. Engelhard MM, Patek SD, Sheridan K, Lach JC, Goldman MD. Remotely engaged: lessons from remote monitoring in multiple sclerosis. Int $J$ Med Inform. 2017;100:26-31. doi:10.1016/j. ijmedinf.2017.01.006

75. Walker UA, Mueller RB, Jaeger VK, et al. Disease activity dynamics in rheumatoid arthritis: patients' self-assessment of disease activity via WebApp. Rheumatology. 2017;56(10):1707-1712. doi:10.1093/ rheumatology/kex229

76. Lalloo C, Stinson JN, Brown SC, Campbell F, Isaac L, Henry JL. PainQuILT: assessing clinical feasibility of a web-based tool for the visual selfreport of pain in an interdisciplinary pediatric chronic pain clinic. Clin J Pain. 2014;30(11):934-943. doi:10.1097/AJP.0000000000000049

77. Catarinella FS, Bos WH. Digital health assessment in rheumatology: current and future possibilities. Clin Exp Rheumatol. 2016;34(101):2-4. 


\section{Publish your work in this journal}

Patient Related Outcome Measures is an international, peer-reviewed, open access journal focusing on treatment outcomes specifically relevant to patients. All aspects of patient care are addressed within the journal and practitioners from all disciplines are invited to submit their work as well as healthcare researchers and patient support groups.
The manuscript management system is completely online and includes a very quick and fair peer-review system. Visit http://www. dovepress.com/testimonials.php to read real quotes from published authors. 